\title{
Time Course of the Development of Motor Behaviors in the Zebrafish Embryo
}

\author{
Louis Saint-Amant, ${ }^{1}$ Pierre Drapeau ${ }^{1,2}$ \\ ${ }^{1}$ Department of Biology, McGill University and Centre for Research in Neuroscience, Montreal \\ General Hospital Research Institute, 1650 Cedar Avenue, Montreal, Quebec H3G 1A4, Canada \\ 2 Department of Neurology and Neurosurgery, McGill University and Centre for Research in \\ Neuroscience, Montreal General Hospital Research Institute, 1650 Cedar Avenue, Montreal, Quebec \\ H3G 1A4, Canada
}

Received 6 March 1998; accepted 25 June 1998

\begin{abstract}
The development and properties of locomotor behaviors in zebrafish embryos raised at $28.5^{\circ} \mathrm{C}$ were examined. When freed from the chorion, embryonic zebrafish showed three sequential stereotyped behaviors: a transient period of alternating, coiling contractions followed by touch-evoked rapid coils, then finally, organized swimming. The three different behaviors were characterized by video microscopy. Spontaneous, alternating contractions of the trunk appeared suddenly at $17 \mathrm{~h}$ postfertilization (hpf), with a frequency of $0.57 \mathrm{~Hz}$, peaked at $19 \mathrm{hpf}$ at $0.96 \mathrm{~Hz}$, and gradually decreased to $<0.1 \mathrm{~Hz}$ by $27 \mathrm{hpf}$. Starting at 21 hpf, touching either the head or the tail of the embryos resulted in vigorous coils. The coils accelerated with development, reaching a maximum speed of contraction before $48 \mathrm{hpf}$, which is near the time of hatching. After
\end{abstract}

27 hpf, touching the embryos, particularly on the tail, could induce partial coils (instead of full coils). At this time, embryos started to swim in response to a touch, preferentially to the tail. The swim cycle frequency gradually increased with age from $7 \mathrm{~Hz}$ at $27 \mathrm{hpf}$ to $28 \mathrm{~Hz}$ at 36 hpf. Lesions of the central nervous system rostral to the hindbrain had no effect on the three behaviors. Lesioning the hindbrain eliminated swimming and touch responses, but not the spontaneous contractions. Our observations suggest that the spontaneous contractions result from activation of a primitive spinal circuit, while touch and swimming require additional hindbrain inputs to elicit mature locomotor behaviors. () 1998 John Wiley \& Sons, Inc. J Neurobiol 37: 622-632, 1998

Keywords: contractions; touch response; lesion; hindbrain; spinal cord; swimming
An important aspect of neurobiology is to understand the development of locomotion. Higher vertebrates pose several problems to these studies because circuits are complex even at the initial stages, and the behaviors themselves are often difficult to characterize. Zebrafish embryos are well suited for studies of development. They can be obtained in large numbers and are easily removed from the chorion, facilitating

Correspondence to: P. Drapeau

Contract grant sponsor: MRC of Canada

Contract grant sponsor: FRS of Quebec

Contract grant sponsor: NSERC of Canada

(C) 1998 John Wiley \& Sons, Inc. CCC 0022-3034/98/040622-11 the analysis of behavior throughout embryonic development. In addition, they are transparent, which simplifies morphological studies. In a wide variety of vertebrates, two key areas underlying locomotion are the hindbrain and spinal cord (Grillner et al., 1997). In the zebrafish, these areas have a simple architecture in that the hindbrain is segmented into eight rhombomeres and the trunk, containing the spinal cord, is divided into about 30 discrete myotomal segments. In the embryo, a small number of identifiable neurons are present in these areas (see below), suggesting that a cellular definition of the locomotor circuits may be possible in this preparation. Furthermore, genetic 
screens have revealed numerous mutations with selective effects on locomotion (Granato et al., 1996), offering the possibility of characterizing the molecular determinants for the development of locomotion.

Aspects of the development of locomotion have been described in vertebrates including Xenopus (Kahn and Roberts, 1982), chick (O’Donovan, 1989), rats (Kudo and Yamada, 1987), and zebrafish (Kimmel et al., 1974). However, the earliest events, including the genesis of motor functions, their maturation during embryonic development, and the nature of the neural networks, are less well characterized. Previous studies of the zebrafish looked at general aspects of the early behaviors, particularly in larvae (Eaton and Farley, 1973; Kimmel et al., 1974). After 2 days of development, newborn larvae vigorously react to touch and show sustained swimming episodes, demonstrating that these behaviors are acquired at some point during embryonic development. More recent work has characterized the development of identifiable neurons in the spinal cord (Myers et al., 1986; Bernhardt et al., 1990; Kuwada et al., 1990) and reticulospinal neurons in the hindbrain of the zebrafish embryo (Kimmel et al., 1981; Mendelson, 1985, 1986; Metcalfe et al., 1990). Despite this wealth of information about central neurons, their relationship to the early locomotor behaviors is unclear, in part because the development of embryonic behaviors has yet to be characterized in detail.

An additional complication in relating neural properties to locomotor behavior during development of the zebrafish is that these two aspects have so far been studied at different temperatures. The lower temperature used in earlier behavioral studies $\left(25^{\circ} \mathrm{C}\right)$ results in a slower rate of growth (Kimmel et al., 1995) than that during morphological investigations (at the now standard temperature of $28.5^{\circ} \mathrm{C}$ ), preventing a direct comparison of the two sets of observations.

To relate the development of behaviors to the growth of neurons, we believed it essential that the two be thoroughly characterized in embryos at the same stages of development. This study describes the sequential appearance of three distinct locomotor behaviors in embryos raised at $28.5^{\circ} \mathrm{C}$. Lesions of different brain areas were also performed to determine the general locus of the neuronal networks necessary for each behavior.

\section{MATERIALS AND METHODS}

\section{Animals}

Adult zebrafish from a local pet store (Danio rerio) and the Longtail variant (from M. Granato, University of Pennsyl- vania) were used as a breeding stock. Adult zebrafish and embryos were kept at $28.5^{\circ} \mathrm{C}$ and cared for as described previously (Westerfield, 1995). Embryos were dechorionated with fine forceps 5 min prior to their use and were kept in an embryo solution of low tonicity containing (in millimoles): $\mathrm{NaCl}, 13.7 ; \mathrm{NaHCO}_{3}, 4.1 ; \mathrm{MgSO}_{4}, 1.0 ; \mathrm{CaCl}_{2}$, $0.98 ; \mathrm{KCl}, 0.54 ; \mathrm{KH}_{2} \mathrm{PO}_{4}, 0.044 ; \mathrm{Na}_{2} \mathrm{HPO}_{4}, 0.025 \mathrm{mM}$; adjusted to $\mathrm{pH} 7.2$, throughout the experiment (Westerfield, 1995). Care was taken to keep the embryos at $28.5^{\circ} \mathrm{C}$ until immediately before the experimental procedures. The solution containing the embryos was transferred to a petri dish and kept at room temperature. This resulted in a gradual cooling by $2^{\circ} \mathrm{C}$ to $3^{\circ} \mathrm{C}$ during the 5 -min period required for most observations and was without noticeable effect on any of the behaviors. After longer time periods when the dish had slowly equilibrated to room temperature, only the spontaneous activity (and neither touch responses nor swimming) was noticeably affected, in that the contraction frequency decreased by $40 \%$. Care was therefore taken to complete the measurements within $5 \mathrm{~min}$, and thus avoid the effect of cooling.

\section{Video Analysis of Behavior}

The movements of the embryos were filmed using a CCD camera (Panasonic wv-bp510) mounted on a dissection microscope. Video fields (two per frame) were recorded on a VCR (Panasonic S-VHS), which was sufficient to resolve the direction, speed, and duration of slow, spontaneous contractions. For the resolution of fast movements, e.g., during touch responses, the specimen was illuminated by a strobe light set to $250 \mathrm{~Hz}$ (Strobotac 1531-A; General Radio Co.), permitting the recording of four superimposed images (separated by $4 \mathrm{~ms}$ ) and therefore a time resolution about four times faster than conventional video. Tail movements were measured by examining individual fields for displacement of the tip of the tail relative to the major body axis.

When the animals had to be restrained for stimulation and filming, they were first anesthetized in a solution containing 0.01\% MS222 (Sigma). Low-melting-point $\left(26^{\circ} \mathrm{C}\right)$ agarose (0.5-1.0\%; Gibco BRL) was added to a separate vial containing embryo medium with MS222 and dissolved by heating to $50^{\circ} \mathrm{C}$ in a water bath. The dissolved agarose was then cooled to $30^{\circ} \mathrm{C}$. Embryos were added to the agarose solution and transferred into the experimental dish. The agarose was then allowed to congeal. To observe movements of the embryo, a hole was cut in the agarose to free only the tail. Anesthetic-free solution at $28.5^{\circ} \mathrm{C}$ was added over the agarose layer and the embryos were allowed to recover (within a few minutes). Neither the anesthetic nor the agarose embedding had noticeable deleterious effects on the embryos or their behaviors.

Responses to touch were evoked by fixing a fine glass probe (a flamed microelectrode) to an audio speaker (1 inch in diameter) to which we applied a square voltage pulse of 1-ms duration. The voltage pulse controlled the extent of the displacement of the probe and was adjusted so that a reliable contraction was elicited in response to the touch. The em- 


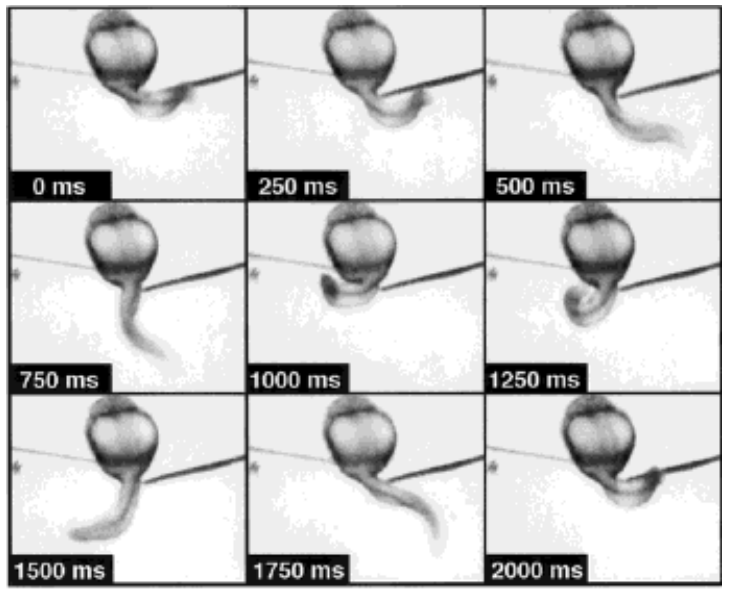

Figure 1 Example of one cycle of spontaneous contractions of an agarose-restrained embryo at $19 \mathrm{hpf}$. Images were captured every 15 frames $(250 \mathrm{~ms})$. The embryo was dorsal side up with the head facing the top of the image. The behavior consisted of a slow contraction to the left followed by a contraction to the right. The length of the embryo was $1.4 \mathrm{~mm}$.

bryos were touched either on the nose or on the tip of the tail, as indicated.

\section{Lesions and Pharmacology}

Transections of the central nervous system (CNS) were performed on anesthetized embryos using a sliver from a razor blade. The embryos were cut at various levels between the forebrain and the spinal cord. In some experiments all neural tissue rostral to the lesion was removed. Postlesion behaviors were either observed directly or videotaped for later analysis. For pharmacological testing, neuromuscular blockers were injected in the head, spinal cord, or heart of agarose-immobilized, anesthetized embryos using a sharp micropipette, and a pressure pulse was applied via a Picospritzer (General Valve). The quantity of solution injected was monitored by using $1 \%$ of the vital dye Fast green (Sigma) in all solutions. The blockers used were $d$-tubocurarine $(100 \mu M$ in $0.9 \% \mathrm{NaCl})$ and $\alpha$-bungarotoxin $(40 \mu M$ in $0.9 \% \mathrm{NaCl})$. In both cases, a control injection was done in other embryos, using the same saline solution without the drug. When immobilization of embryos was needed for an experiment, we used $0.01 \%$ MS222 (Sigma).

\section{RESULTS}

Zebrafish embryos showed no movement until 17 hours postfertilization (hpf); after this time, three sequential behaviors appeared (spontaneous contractions, touch responses, and swimming) that will be described in the order of their appearance during development of the embryo.

\section{Spontaneous Contractions}

The first movements observed in the embryos started precisely at $17 \mathrm{hpf}$ and consisted of alternating sideto-side contractions of the tail, much like a metronome. The contractions consisted of slow coils during which the tip of the tail reached the head but where only the initial segments appeared to be active. This behavior could be observed in intact eggs, but was easier to characterize upon freeing the embryos from the chorion by using fine forceps. Figure 1 shows successive images (taken every $250 \mathrm{~ms}$ ) of a 19-hpf embryo embedded in agarose with the tail free to move. As can be seen, one complete cycle of two alternating contractions lasted $2 \mathrm{~s}$, corresponding to a contraction frequency of $1 \mathrm{~Hz}$. Although dechorionation increased the frequency of contractions, particularly at the earliest times, it had no effect on the time of appearance or on the time of peak activity and subsequent decline.

The spontaneous behavior reached a peak frequency of $0.96 \pm 0.06 \mathrm{~Hz}$ ( $n=30$ embryos; from six batches of eggs) at $19 \mathrm{hpf}$, then gradually declined over the course of several hours to $0.08 \pm 0.02 \mathrm{~Hz}(n$ $=25$ embryos; four batches) by 26 hpf (Fig. 2). The regularity of spontaneous activity changed with de-

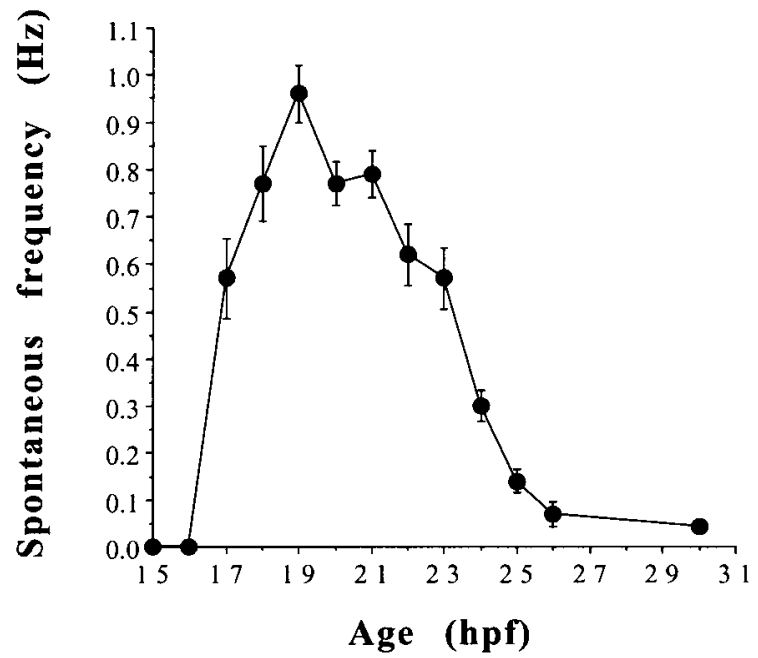

Figure 2 Development of spontaneous contractions. Each data point represents the average frequency of contractions and standard errors for all freely moving embryos examined at each time point. Measurements were performed on four to eight embryos per batch, and two to six batches were used per time point. Average $n$ per time interval $=16$; range, $8-44$. 


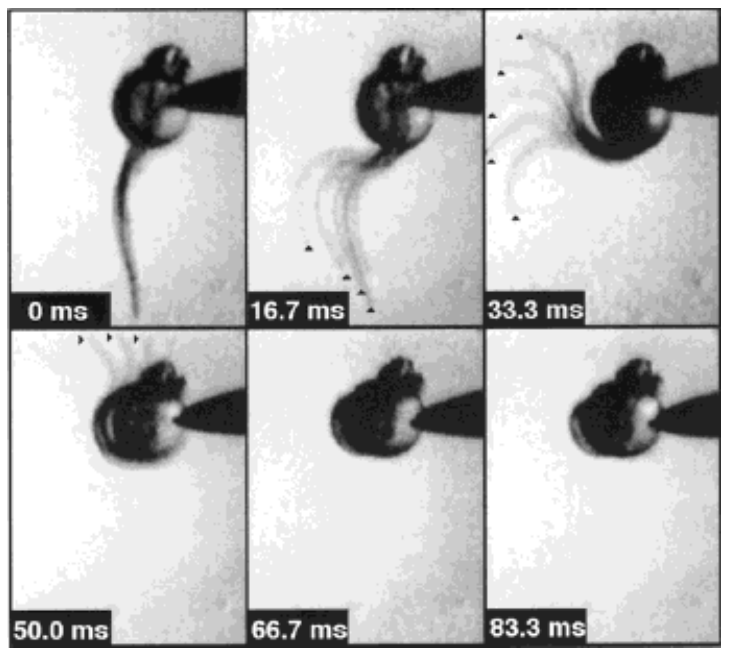

Figure 3 Example of a touch response at $25 \mathrm{hpf}$ in a freely moving embryo. Each successive tail image is separated by $4 \mathrm{~ms}$. For clarity, arrowheads point to the tip of the tail. In this example, the embryo is dorsal side up with the rostral end facing the top of the image. Note that the embryo held its contracted position during the last three frames. The total length of the embryo was $1.9 \mathrm{~mm}$.

velopment. The very young embryo (18-23 hpf) contracted continuously, much like a windshield wiper, whereas the older embryos ( $>24 \mathrm{hpf}$ ) showed bursts of three to five coils often followed by periods of inactivity that could last over $20 \mathrm{~s}$.

\section{Response to Touch}

When 1-day-old embryos were touched either on the head or the tail, they usually reacted with contralateral contractions resembling a faster version of the coils described above. Figure 3 shows successive images of a freely moving embryo at $25 \mathrm{hpf}$, captured at a normal video field rate of $60 \mathrm{~Hz}$ (16.7 ms apart). To enhance the resolution of rapid coils, the embryo was illuminated with a strobe light set at $250 \mathrm{~Hz}$, which permitted the capture of up to five superimposed images separated by $4 \mathrm{~ms}$ (shown by arrowheads at the tips of the tails). The strobe light enabled us to assess tail displacements at 4-ms intervals and to make measurements of contraction speed.

The responses to touching the head were rapid coils (Fig. 3, lower panels) followed by a slower relaxation. Up to three of these alternating coils were often seen in response to a single touch. Note that the contraction (to the left) is contralateral to the touch (to the right). These touch responses were first observed at $21 \mathrm{hpf}, 2 \mathrm{~h}$ after the peak of spontaneous contractions, and were followed by swim episodes at later times (after $27 \mathrm{hpf}$ ). Before the onset of swimming, touching the head or tail elicited similar behaviors; once swimming could be elicited, touching the tail resulted in a modified touch response. Here, we describe the responses to touching the head. The swim behavior and the associated alteration in the response to touching the tail will be described below.

At the peak of spontaneous activity (Fig. 2) (19 hpf), the embryos were unresponsive to touch, since asynchronous tactile stimulation did not interfere with the spontaneous behavior. By $21 \mathrm{hpf}$, the embryos started to show a response to touch. To determine more precisely if the embryos were indeed responding to touch, or if they were coiling spontaneously, embryos were immobilized in agarose with the tail free to move. [We noticed a small but insignificant ( $p$ $>$.1) decrease in the peak rate of spontaneous contractions (19 hpf) when the embryos were held in agarose compared to the free-swimming embryos as the frequency went from $0.96 \pm 0.06 \mathrm{~Hz}$ (Fig. 2) to $0.75 \pm 0.10 \mathrm{~Hz}$ (Fig. 4)]. The embryos were stimulated by touching their heads repetitively at two different rates, 1 and $2 \mathrm{~Hz}$. If the embryos responded to these stimuli, they were expected to follow these rates

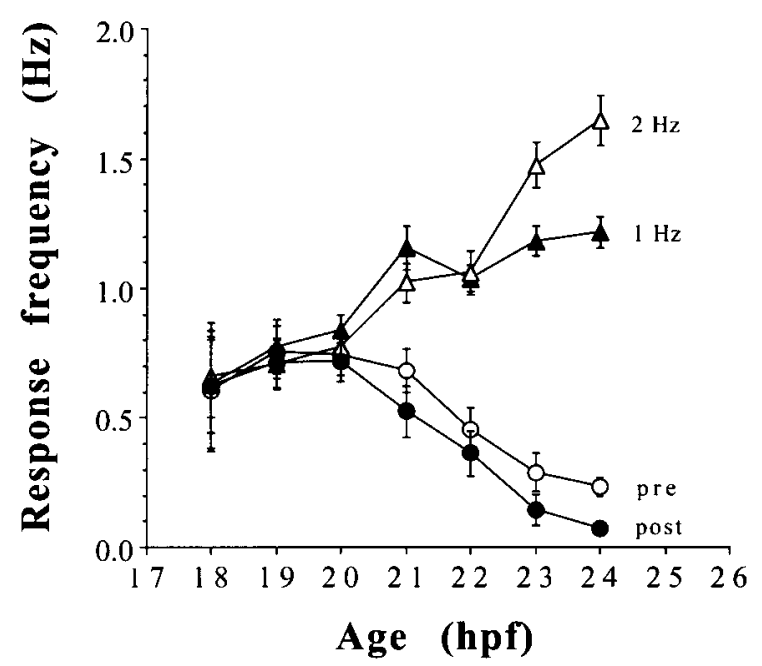

Figure 4 Onset of the touch response. The onset of the touch response was evaluated for each agarose-restrained embryo tested by comparing the contraction frequency during repetitive stimulation (at 1 or $2 \mathrm{~Hz}$ ) to the frequency during control periods (without repetitive stimulation) at different times of development, as described in the text. The open circles are the results for prestimulation controls, the closed circles are poststimulation controls, the closed triangles are $1-\mathrm{Hz}$ stimulation and the open triangles are $2-\mathrm{Hz}$ stimulation. Error bars represent standard error. Measurements were performed on three to five embryos per batch, and one to three batches were used per time point. Average $n$ per time interval $=8$; range, $3-13$. 


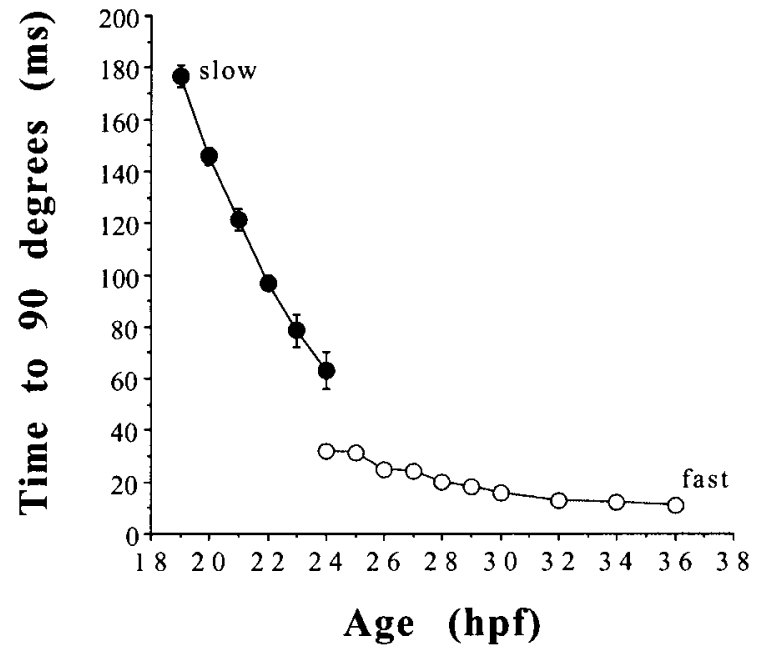

Figure 5 Time for spontaneous and evoked contractions. The time taken for the tail to bend from rest to $90^{\circ}$ relative to head axis was measured at hourly intervals ( $n$ ranging from 6 to 55; from one to six batches) in freely moving embryos. The closed circles are for spontaneous contractions and the open circles are for touch-evoked contractions. All data points have error bars (S.E.).

of stimulation, rather than decline in frequency, as was seen during the spontaneous contractions. By comparing the frequency of contractions during stimulation with the frequency of contractions when not stimulated, the time of onset of touch responses could be determined. Each embryo used in Figure 4 was filmed for 5 min during which time a protocol consisting of the following steps was used (1 min duration each): (a) no stimulation ("pre," open circles), (b) 1-Hz stimulation (closed triangles), (c) no stimulation (not shown), (d) 2-Hz stimulation (open triangles), and (e) no stimulation ("post," closed circles). No difference in contraction frequency between control and stimulated embryos was found until $21 \mathrm{hpf}$, at which time the stimulations elicited significantly higher rates of contraction $(1.16 \pm 0.09 \mathrm{~Hz})$ than the controls $(0.68 \pm 0.08 \mathrm{~Hz} ; p<.001)$ (Fig. 4). This difference became more obvious at later times (measured up to $24 \mathrm{hpf}$ ) as the frequency of the spontaneous contractions continued to decline, whereas the stimulated activity increased in frequency. Similar results were obtained when the above-mentioned protocol was changed to touching the tail of the embryos (not shown). Also observed was a decline in the responses to higher-frequency stimulation, suggesting habituation, but we did not characterize this further.

The speed of the first coil in response to touching the head was seen to increase dramatically during development. To compare the rates of contraction at different ages, the time taken for the tip of the tail to bend from the resting position to $90^{\circ}$ relative to the head axis was measured. This estimate yielded a mean contraction time of $32.0 \pm 1.8 \mathrm{~ms}(n=5)$ at $24 \mathrm{hpf}$ and declined progressively to $10.6 \pm 0.4 \mathrm{~ms}(n=38$, four batches) at $36 \mathrm{hpf}$ (Fig. 5, open circles). The mean contraction time for spontaneous coils was estimated (Fig. 5, closed circles). At the peak (19 hpf) of spontaneous activity, the contraction time was 177 $\pm 4 \mathrm{~ms}$ ( $n=22$, two batches) and it decreased to 63 $\pm 7 \mathrm{~ms}$ ( $n=9$, two batches) at $24 \mathrm{hpf}$. Therefore, the touch-evoked coils at $24 \mathrm{hpf}$ were twice as fast as the spontaneous coils, demonstrating a further difference between these behaviors.

\section{Swimming}

The third behavior to appear in the early embryo was swimming, which was a new behavior that started in response to touch after $26 \mathrm{hpf}$ and resulted in the net forward movement of the embryo by at least one body length. Embryos with their heads restrained in agarose (to obtain clearer images) showed sustained swimming movements of the tail that were of lower amplitude (i.e., the tail contracted by $<60^{\circ}$ on each side) (Fig. 6) and higher frequency [Fig. 7(A)] than the larger and less frequent contractions during spontaneous activity. The cyclic contraction frequency during swimming evoked by a tail touch on a free swimming embryo was already $7.3 \pm 0.5$ cycles/s (one cycle $=$ two contractions) when first observed (at $26 \mathrm{hpf}$ ) [Fig. 7(A)] ( $n=9$, one batch), which is about 17 times faster than the highest frequency of spontaneous contractions. The frequency of swimming increased

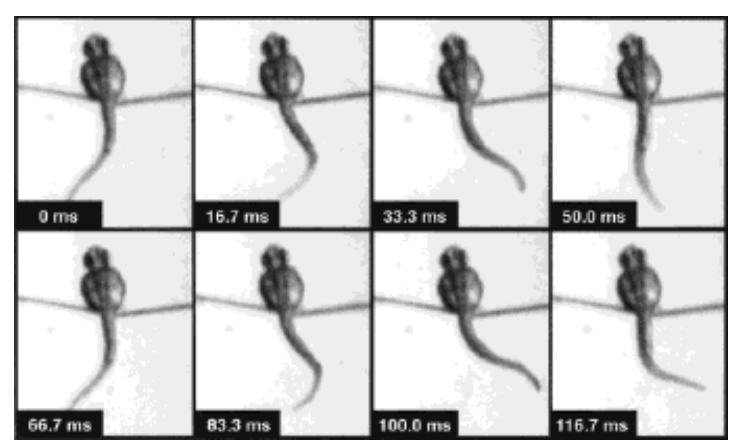

Figure 6 The swimming behavior at $30 \mathrm{hpf}$. Swimming can be seen as a sequence of alternating, low-amplitude contractions of the tail occurring at a high frequency. In this example, one full cycle of swimming is observed from 0 to $66.7 \mathrm{~ms}$; if maintained throughout an episode, this rhythm would yield an average cycle frequency of $15 \mathrm{~Hz}$. The embryo was immobilized in agarose, dorsal side up and anterior to the top of the image. The length of the embryo was $2.3 \mathrm{~mm}$. 

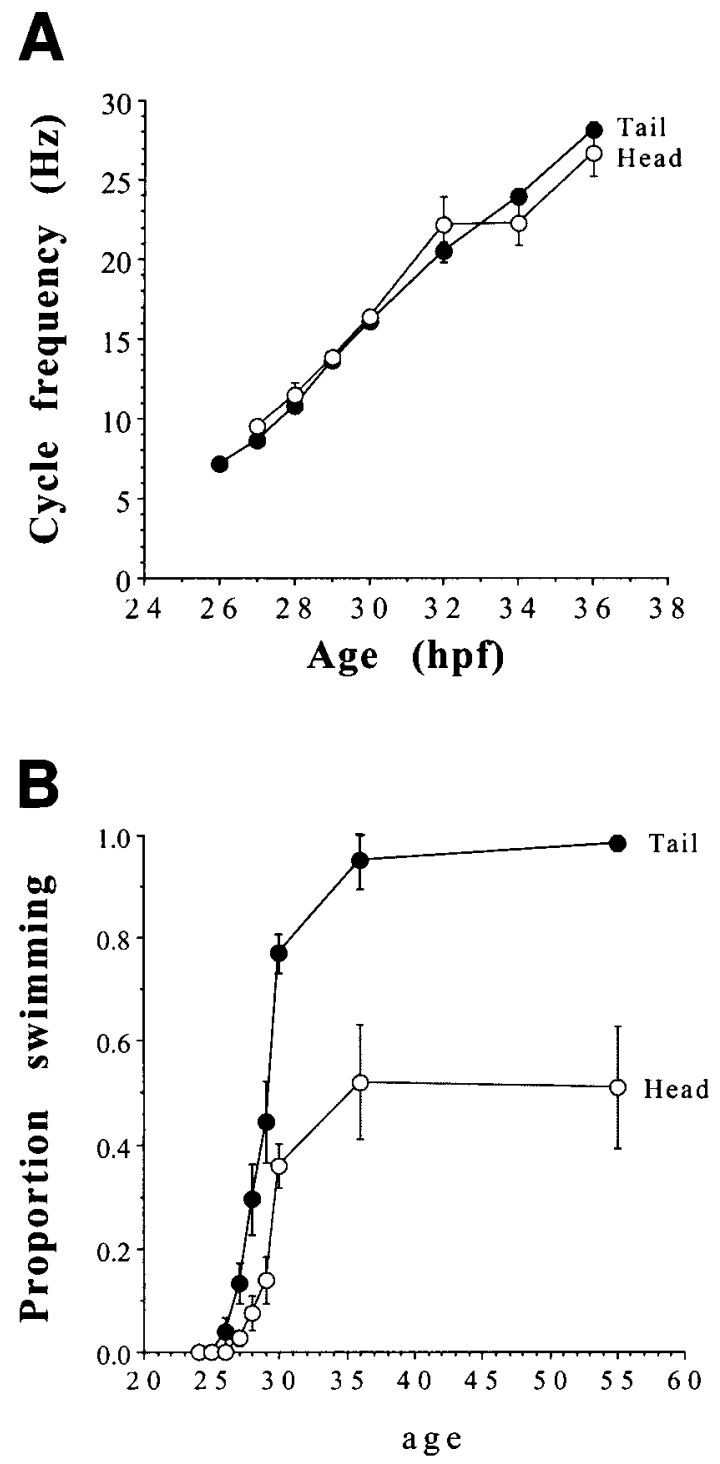

Figure 7 Cycle frequency and proportion of embryos showing swimming. (A) Change in cycle frequency of swimming with development. A linear increase in frequency is seen up to $36 \mathrm{hpf}$. (B) Proportion of embryos swimming after a tactile stimulus either on the tail (filled circles) or on the head (open circles). All data points have error bars (S.E.). Range of $n=5-17$ embryos, from two to three batches.

linearly during development up to $28.7 \pm 0.5$ cycles/s by 36 hpf [Fig. 7(A)] ( $n=6$, two batches; regression analysis for the swimming cycle frequency versus hpf yields: head touch $R^{2}=0.962$, tail touch $R^{2}$ $=0.997)$.

The sudden onset of swimming, as with the other behaviors, suggests that the development of the underlying circuitry may be independent of experience-in other words, "hardwired." In zebrafish, blocking neural activity with tetrodotoxin (TTX) was shown to have no effect on retino-tectal projections (Kaethner et al., 1994). To examine the role of locomotor activity during the development of swimming, newly laid eggs were anesthetized in $0.01 \%$ MS-222 for up to several days. During this period, locomotor activity was virtually abolished in that only by careful observation of large numbers of eggs over periods of many minutes could we detect an occasional contraction. The onset of pigmentation, normally around 26 hpf (Kimmel et al., 1995), was delayed by 2-3 h. Furthermore, embryos dechorionated at $30 \mathrm{hpf}$ in an anesthetic-free solution recovered their responsiveness to touch within a few minutes but did not yet swim, whereas normal embryos start swimming after 26 hpf [Fig. 7(A)]. These observations can be explained most simply if the anesthetic caused a general delay in development of a few hours. If left in anesthetic for several days, the embryos failed to hatch. When dechorionated after $48 \mathrm{hpf}$, the embryos swam in response to a touch, but they swam in small circles. Unanesthetized embryos sometimes swim in circles for a short time after dechorionation (5-10 $\mathrm{min})$, but this circling stops when the tail becomes straight at rest, whereas anesthetized embryos only recovered after a few hours. This suggests that embryonic contractions are necessary to maintain a straightened tail but are not necessary for the establishment of the swim circuitry.

Swimming was elicited more readily as the embryos aged and was observed more often when the embryos were touched on the tail [Fig. 7(B)]. By 36 hpf, a significantly higher fraction of tail stimuli ( 95 $\pm 6 \% ; n=185$; seven batches) resulted in a swimming episode compared to head stimuli $(52 \pm 11 \%$; $n$ $=181$; seven batches; $t$ test at $36 \mathrm{hpf} ; p<.001$ ). The preferential induction of swimming by tail stimuli led us to examine more closely the properties of the touch response at later stages of development. Before the onset of swimming (as described above), embryos responded to head and tail stimuli with the same behavior: full coils, which reoriented the embryo away from the stimulus [Fig. 8(A)]. Once swimming could be elicited, a partial response was evoked, specifically when touching the tail [Fig. 8(B,C); $\chi^{2} p$ $<.01]$. The partial response was subtler, as it consisted of the tail bending toward but not reaching over the head; the embryo was displaced momentarily but was not reoriented. Although individual episodes of swimming could arise following either full or partial coils, it was clear that when the embryos were unable to swim, they almost always showed full coils, as only rarely did we observe partial coils [Fig. 8(C)] $(26$ hpf). The appearance of partial coils in association 


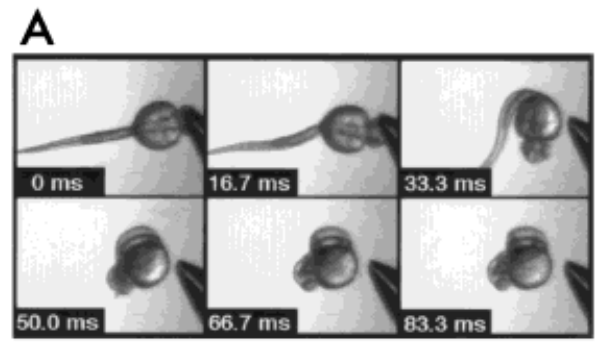

B
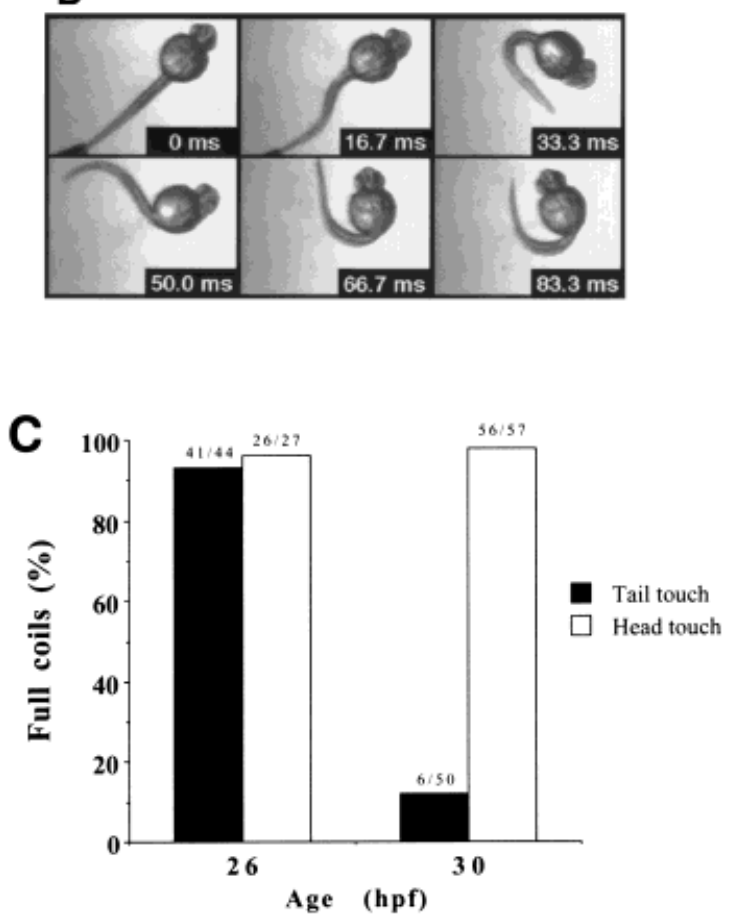

Figure 8 Head and tail stimuli cause different behaviors in the same 30-hpf embryo. (A) Normal coiling response to head stimuli. Note the turning of the embryo, which held its contracted position from 50 to $83.3 \mathrm{~ms}$. (B) Partial coil in response to a tail touch $(0-33.3 \mathrm{~ms})$, which was followed by a second partial coil $(50-83.3 \mathrm{~ms})$, and finally a swim episode (not shown). The length of the embryo was $2.1 \mathrm{~mm}$. (C) Percentage of the embryos showing full coils in response to touching the tail (open bars) or head (closed bars) at 26 and $30 \mathrm{hpf}$. The numbers above each bar are the actual fractions of embryos tested that showed full coils.

with the appearance of swimming suggests that the two may be related.

\section{Effects of Lesions on Locomotor Behaviors}

A neural origin for embryonic locomotor behaviors was demonstrated by the inhibition of contractions upon injection of the embryos with the cholinergic blockers $\alpha$-bungarotoxin and $d$-tubocurarine, which suppress neuromuscular transmission in the zebrafish (Grundwald et al., 1988). These results were verified and a blockade of all locomotor behaviors including the spontaneous contractions was observed (not shown).

To determine which regions of the CNS play a role in generating the three embryonic behaviors, the effects of lesions were studied. The CNS was transected at different levels and at different stages of development. The time of analysis was chosen for clear identification and robustness of the behaviors. The effects of lesions were studied around the peak of activity (19-22 hpf) for spontaneous contractions, after $25 \mathrm{hpf}$ for touch-evoked responses and at 34-38 hpf for swimming. Even though these lesions seem drastic, the health of the embryos was not compromised for several hours afterward, longer than the period of observation. This is perhaps not surprising, as spontaneous contractions and touch responses developed prior to blood flow, which was minimal at the time that swimming appeared. A limited amount of necrotic tissue was observed close to the site of the lesions but no further than $50 \mu \mathrm{m}$ away. All the lesioned embryos were included in the data sets (Fig. 9 ); i.e., there was no need to remove some of the lesioned embryos from the data set owing to poor health or death.

Lesions that were rostral to the hindbrain had no effect on any of the behaviors. In fact, removal of all neural tissue rostral to the hindbrain was also without effect; i.e., the entire behavioral repertoire was retained when only the hindbrain and spinal cord were left intact. Lesions of the hindbrain and spinal cord affected the behaviors differently. As shown in Figure 9(A), lesions of the hindbrain had no effect on the spontaneous contractions. However, as the lesions were progressively made caudally along the spinal cord, the behavior was gradually suppressed and was completely stopped for lesions beyond the sixth somite.

Hindbrain lesions did affect touch responses and swimming [Fig. 9(B,C)]. In an effort to more clearly define the role of the hindbrain, we attempted to lesion it at various locations. However, the hindbrain lesions were hard to localize because it is more uniform at these early stages and the only consistent landmark is the otolith midway along the hindbrain. The hindbrain lesions were therefore grouped into three categories: rostral, in which the otolith was left intact but the border with the midbrain was damaged; middle, in which the region near the otolith was damaged; and caudal, in which the otolith was le- 

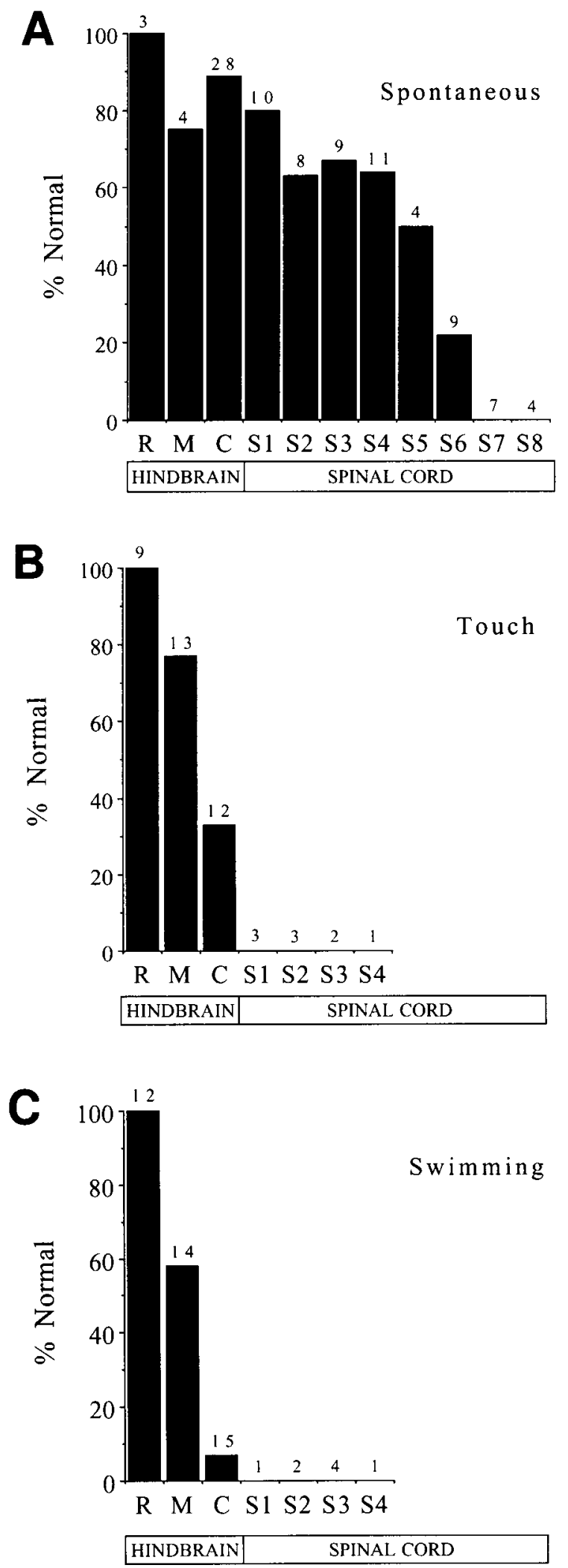

Figure 9 The effect of lesions on the early locomotor behaviors. The graphs display the percentage of embryos showing a normal behavior versus the location of the lesion, sioned but the border with the spinal cord was intact. More caudal lesions of the hindbrain had more dramatic effects on touch responses and swimming, and both responses were eliminated upon complete removal of the hindbrain.

\section{DISCUSSION}

We found that the three locomotor behaviors of the zebrafish embryo appeared at highly precise stages during a 10-h period and reached a larval level of development by $36 \mathrm{hpf}$, which is $12 \mathrm{~h}$ prior to the earliest hatchings. Furthermore, the suppression of movements in anesthetized embryos did not appear to affect the development of swimming. Together with the observation of mutations that can disrupt locomotion (Granato et al., 1990), this is consistent with a precise developmental program during the onset and maturation of embryonic locomotor behaviors.

\section{Spontaneous Contractions}

The spontaneous behavior started suddenly at $17 \mathrm{hpf}$, with a peak at $19 \mathrm{hpf}$, followed by gradual decline over the next few hours. The spontaneous contractions never completely stopped and are likely the eventual cause of hatching (Kimmel et al., 1974). A similar peak in the frequency of spontaneous contractions was reported by Kimmel et al. (1974) for embryos raised at $25^{\circ} \mathrm{C}$, except that the peak was delayed until 22-23 hpf. This delay in spontaneous activity is presumably due to the general slowing of development in embryos raised at lower temperatures (Kimmel et al., 1995). We also observed that spontaneous contractions were unaffected by lesions rostral to the spinal cord, demonstrating that the neural substrates for this behavior are limited to the spinal cord. As spontaneous limb movements are also observed in the chick embryo (Alconero, 1965) and are mediated by spinal neurons (Ho and O'Donovan, 1993), the spinal cord appears to be the locus for precocious development of vertebrate locomotor functions.

and the numbers at the top of each bar are the number of experiments. (A) Results for the spontaneous behavior. (B) Results for the touch response. (C) Effects of lesions on swimming. R, M, and C represent the rostral, middle (near the otholith), and caudal part of the hindbrain, respectively; S1-S8 are the successive somites of the tail starting from the hindbrain. Forebrain lesions had no effect on early behaviors, whereas lesion of the hindbrain affected the touch and swimming but not the spontaneous behavior. 
Very few cells with axons are present in the spinal cord at this stage of development in zebrafish embryos reared at $28.5^{\circ} \mathrm{C}$. At the efferent side, the most caudal of the three primary motoneurons in each hemisegment along the spinal cord is known to project to the muscles by $18 \mathrm{hpf}$, and the two other primary motoneurons project within a few hours (Myers et al., 1986). Along the spinal cord, only five other types of neurons (with only one or two homologs of each per hemisegment) have extended axons at that time in development: the Rohon-Beard sensory neurons, the ventral longitudinal descending interneurons (VeLD), the circumferential descending interneuron (CiD), the dorsal longitudinal ascending interneurons (DoLA), and the commissural primary ascending interneurons (CoPA) (Bernhardt et al., 1990; Kuwada et al., 1990). Rohon-Beard cells start projecting axons at $15 \mathrm{hpf}$, and by the time the spontaneous behavior starts, the axons have crossed several somitic boundaries (Kuwada et al., 1990). However, the embryos appeared to be insensitive to touch before $21 \mathrm{hpf}$, suggesting that Rohon-Beard neurons do not participate in generating spontaneous contractions. In the Xenopus embryo, interneurons resembling the VeLD and $\mathrm{CiD}$ have been shown to produce a tonic drive, whereas commissural interneurons resembling CoPA provide the midcycle inhibition seen in motoneurons during swimming, and together constitute the spinal central pattern generator (Roberts, 1990). Similar spinal interneurons may play a comparable role in adult goldfish (Fetcho, 1992). VeLD and CiD neurons may thus be excitatory, whereas CoPA neurons may be inhibitory during the cyclical, spontaneous contractions if these are an early manifestation of the swim circuitry. It therefore seems that a small number of identifiable neurons underlie this behavior, providing a conceptual framework for future cellular neurophysiology.

\section{Touch Response}

Starting at $21 \mathrm{hpf}$, tactile stimuli resulted in rapid tail coils. Although previous work (Eaton et al., 1981) has characterized in detail the properties of this behavior in larvae, we were surprised to see that it appeared as early as $21 \mathrm{hpf}$ in the embryo. There is no addition of new cells bearing processes in the spinal cord between 18 and 22 hpf (Kuwada et al., 1990). Therefore, the touch response must be mediated by newly functional sensory inputs that ultimately result in activation of the primary motoneurons. Perception of head stimuli is mediated by trigeminal neurons (Kimmel et al., 1990), whereas in the tail, Rohon-Beard neurons are the only sensory neurons present (Kuwada et al., 1990; Metcalfe et al., 1990).
Our lesion experiments indicated that in addition to the spinal cord, an intact hindbrain was essential for the touch response. Interestingly, both trigeminal and Rohon-Beard neurons project to the hindbrain where they fasciculate, and the latter are known to contact reticulospinal neurons such as the giant Mauthner cell at $\sim 19 \mathrm{hpf}$ (Kimmel et al., 1990). The Mauthner cell sends its axon contralaterally and then caudally toward the spinal cord, where it pioneers the medial lateral fascicle, reaching the third somite by $21 \mathrm{hpf}$, and is therefore the only reticulospinal neuron present at this stage (Mendelson, 1985). Interestingly, the embryonic touch response was contralateral, consistent with a role for the Mauthner cell. Reticulospinal neurons are known to mediate startle responses in teleost fish (Nissanov et al., 1990; Faber and Korn, 1978; Fetcho, 1992) by activating excitatory spinal interneurons and motoneurons. Although the touch response is present in zebrafish larvae (Eaton et al., 1981; Fetcho and O'Malley, 1995) and, as reported here, in the embryo, the startle response is elicited by touch as well as by other sensory modalities which develop only at postembryonic stages (Eaton and Farley, 1973). Our lesion data supports involvement of the hindbrain in the early touch response, but more precise lesion studies such as cellular ablations are needed to address the question of specific reticulospinal neuron involvement. We are nevertheless drawn to the attractive proposition that the early touch response is an immature manifestation of the startle response and may be due to activation of only a few (and perhaps just one pair of) reticulospinal neurons.

Tail stimuli started to elicit a modified, partial response which did not reorient the embryo at the time $(\sim 27 \mathrm{hpf})$ that they evoked swim episodes and did so more reliably than when the head was touched. This would make sense at a behavioral level, because upon tail stimulation, the fish would swim away from the stimulus. The head touches always produced full coils that could turn the embryo. Behaviorally, this would cause the reorientation of the fish away from the head stimulus, followed by swimming and escape. Similar differences in behavior have been observed in the zebrafish larva (Eaton et al., 1984), and our results clearly show that this difference develops early in the embryo.

Based on their observations with goldfish, Foreman and Eaton (1993) proposed that head touches would activate a larger population of reticulospinal neurons and lead to a more massive contraction than touches to the tail, which would activate only the Mauthner cells and produce a smaller response. Activation of different populations of neurons (but always the Mauthner cell) in response to either head or 
tail stimuli has recently been demonstrated by calcium imaging of hindbrain neuronal activity in the zebrafish larva (O’Malley et al., 1996). Although the early embryonic touch response was similar for both head and tail stimuli, by $27 \mathrm{hpf}$ the hindbrain may have further differentiated in that it generates a new touch response, the partial coil, at the time that we observed a new behavior, swimming.

\section{Swimming}

After $27 \mathrm{hpf}$, the embryos started to show episodes of swimming in response to a touch. The rate of swimming increased by $36 \mathrm{hpf}$ to a rate $(30 \mathrm{~Hz})$ comparable to that which can be estimated from electromyographic recordings in adult zebrafish (Liu and Westerfield, 1988), but the embryos were too small for us to confirm the muscle activity patterns. By $27 \mathrm{hpf}$, the spinal cord and hindbrain have increased in their cellular complexity: At least four more types of interneurons extend axons (Bernhardt et al., 1990) and 20 or so secondary motoneurons innervate the musculature (Myers et al., 1986). Interestingly, a mutation that prevents the formation of secondary neurons, including the secondary motoneurons, prevents swimming (Grundwald et al., 1988). Thus, swimming may be limited by the appearance of secondary motoneuron function.

An advantage of the zebrafish embryo for future studies of the development of locomotion is that it may be possible to study the physiology of individually identifiable neurons of the locomotor network in vivo, e.g., by calcium imaging of neuronal populations (Fetcho and O'Malley, 1995; O’Malley et al., 1996). Another advantage is that the study of locomotor genetics (Granato et al., 1996) may permit a combination of cellular and molecular approaches to understanding the development of locomotor circuits.

This work was supported by an MRC of Canada studentship to LS-A and FRS of Quebec Senior Research Scholarship and grant from the NSERC of Canada to PD. The authors thank E. Harvey-Girard and G. Pollack for helpful discussions.

\section{REFERENCES}

Alconero, B. B. (1965). The nature of the earliest spontaneous activity of the chick embryo. J. Embryol. Exp. Morphol. 13:255-266.

Bernhardt, R. R., Chitnis, A. B., Lindamer, L., and KuWADA, J. Y. (1990). Identification of spinal neurons in the embryonic and larval zebrafish. J. Comp. Neurol. 302: 603-616.
Eaton, R. C., and Farley, R. D. (1973). Development of the Mauthner neurons in embryos and larvae of the zebrafish, Brachydanio rerio. Copeia 4:673-682.

Eaton, R. C., Lavender, W. A., and Wieland, C. M. (1981). Identification of Mauthner-initiated response patterns in goldfish: evidence from simultaneous cinematography and electrophysiology. J. Comp. Physiol. 144:521-531.

Eaton, R. C., Nissanov, J., and Wieland, C. M. (1984). Differential activation of Mauthner and non-Mauthner startle circuits in the zebrafish: implications for functional substitution. J. Comp. Physiol. A 155:813-820.

FABER, D. S., and Korn, H. (1978). Neurobiology of the Mauthner Cell. Raven Press, New York.

Fetcho, J. R. (1992). The spinal motor system in early vertebrates and some of its evolutionary changes. Brain Behav. Evol. 40:82-87.

Fetcho, J. R., and O'Malley, D. M. (1995). Visualization of active neural circuitry in the spinal cord of intact zebrafish. J. Neurophysiol. 73:399-406.

Foreman, M. B., and EAton, R. C. (1993). The direction change concept for reticulospinal control of goldfish escape. J. Neurosci. 13:4101-4113.

Granato, M., van Eeden, F. J. M., Schach, U., Trowe, T., Brand, M., Furutani-Seiki, M., Haffter, P., HammerSChmidt, M., Heisenberg, C.-P., Jiang, Y.-J., Kane, D. A., Kelsh, R. N., Mullins, M. C., Odenthal, J., and Nusslein-Volhard, C. (1996). Genes controlling and mediating locomotion behavior of the zebrafish embryo and larva. Development 123:399-413.

Grillner, S., Georgopoulos, A. P., and Jordan, L. M. (1997). Selection and initiation of motor behavior. In: Neurons, Networks, and Motor Behavior. D. Stein, A. Stuart, S. Selverston, and S. Grillner, Eds. MIT Press, Cambridge, MA, pp. 1-19.

Grunwald, D. J., Kimmel, C. B., Westerfield, M., Walker, C., and Streisigner, G. (1988). A neural degeneration mutation that spares primary neurons in the zebrafish. Dev. Biol. 126:115-128.

Ho, S., and O'Donovan, M. J. (1993). Regionalization and inter-segmental coordination of rhythm generating networks in the spinal cord of the chick embryo. J. Neurosci. 13:1345-1371.

Kaethner, R. J., and Stuermer, C. A. (1984). Growth behavior of retinotectal axons in live zebrafish embryos under TTX-induced neural impulse blockade. J. Neurobiol. 25:781-796.

Khan, J. A., and RoberTs, A. (1982). The central nervous origin of the swimming motor pattern in embryos of Xenopus laevis. J. Exp. Biol. 99:185-196.

Kimmel, C. B., Ballard, W. W., Kimmel, S. R., Ullman, B., and Schilling, T. F. (1995). Stages of embryonic development of the zebrafish. Dev. Dynam. 203:253-310.

Kimmel, C. B., Hatta, K., and Metcalfe, W. K. (1990). Early axonal contacts during development of an identified dendrite in the brain of the zebrafish. Neuron 4:535-545.

Kimmel, C. B., Patterson, J., and Kimmel, R. O. (1974). The development and behavioral characteristics of the startle response in the zebrafish. Dev. Psychobiol. 7:47-60. 
Kimmel, C. B., Session, S. K., and Kimmel, R. J. (1981). Morphogenesis and synaptogenesis of the zebrafish Mauthner neuron. J. Comp. Neurol. 198:101-120.

Kuwada, J. Y., Bernhardt, R. R., and Nguyen, N. (1990). Development of spinal neurons and tracts in the zebrafish embryo. J. Comp. Neurol. 302:617-628.

Kudo, N., and Yamada, T. (1987). N-Methyl-D,L-aspartateinduced locomotor activity in a spinal cord-hindlimb muscle preparation of the newborn rat studied in-vitro. Neurosci. Lett. 75:43-48.

Mendelson, B. (1985). Soma position is correlated with time of development in three types of identified reticulospinal neurons. Dev. Biol. 112:489-493.

Mendelson, B. (1986). Development of reticulospinal neurons of the zebrafish. II. Early axonal outgrowth and cell body position. J. Comp. Neurol. 251:172-184.

Metcalfe, W. K., Myers, P. Z., Trevarrow, B., Bass, M. B., and Kimmel, C. B. (1990). Primary neurons that express the L2/HNK-1 carbohydrate during early development in the zebrafish. Development 110:491-504.
Myers, P. Z., Eisen, J. S., and Westerfield, M. (1986). Development and axonal outgrowth of identified motoneurons in the zebrafish. Eur. J. Neurosci. 6:22782289.

Nissanov, J., Eaton, R. C., and DiDomenico, R. (1990). The motor output of the Mauthner cell, a reticulospinal command neuron. Brain Res. 517:88-98.

O'Donovan, M. J. (1989). Motor activity in the isolated spinal cord of the chick embryo: synaptic drive and firing pattern of single motoneurons. J. Neurosci. 9:943-958.

O’Malley, D. M., KaO, Y. H., and Fetcho, J. R. (1996). Imaging the functional organization of zebrafish hindbrain segments during escape behaviors. Neuron 17:1145-1155.

Roberts, A. (1990). How does a nervous system produce behavior? A case study in neurobiology. Sci. Prog. Oxford 74:31-51.

Westerfield, M. (1995). The Zebrafish Book: A Guide for the Laboratory Use of Zebrafish (Brachydanio rerio). University of Oregon Press, Eugene, OR. 